\title{
Artificial intelligence-based myocardial texture analysis in etiological differentiation of left ventricular hypertrophy
}

\author{
Fei Yu' ${ }^{1}$, Haibo Huang ${ }^{2,3}$, Qihui Yu ${ }^{2,3}$, Yuqing Ma ${ }^{1}$, Qi Zhang ${ }^{2,3,4}$, Bo Zhang ${ }^{1}$ \\ ${ }^{1}$ Department of Ultrasound in Medicine, Shanghai East Hospital, Tongji University School of Medicine, Shanghai, China; ${ }^{2}$ Shanghai Institute \\ of Advanced Communication and Data Science, The SMART (Smart Medicine and AI-based Radiology Technology) Lab, Shanghai University, \\ Shanghai, China; ${ }^{3}$ Institute of Biomedical Engineering, School of Communication and Information Engineering, Shanghai University, Shanghai, \\ China; ${ }^{4}$ Shanghai Key Laboratory of Artificial Intelligence for Medical Image and Knowledge Graph, Shanghai, China \\ Contributions: (I) Conception and design: Q Zhang, B Zhang; (II) Administrative support: Q Zhang, B Zhang; (III) Provision of study materials or \\ patients: F Yu, Y Ma; (IV) Collection and assembly of data: F Yu, H Huang, Q Yu, Y Ma; (V) Data analysis and interpretation: F Yu, H Huang, Q Yu; \\ (VI) Manuscript writing: All authors; (VII) Final approval of manuscript: All authors. \\ Correspondence to: Bo Zhang, MD. Department of Ultrasound in Medicine, Shanghai East Hospital, No. 150, Jimo Road, Shanghai 200120, China. \\ Email: zhangbo2016@tongji.edu.cn; Qi Zhang, PhD. Room 803, Xiangying Building, Shanghai University, No. 333, Nanchen Road, Shanghai \\ 200444, China. Email: zhangq@t.shu.edu.cn; zhangq@shu.edu.cn.
}

Background: Transthoracic echocardiography (TTE) is widely used in clinics to evaluate left ventricular hypertrophy (LVH). However, TTE is usually insufficient for the etiological diagnoses when morphological and functional features are nonspecific. With the booming of computer science and artificial intelligence (AI), previous literature has reported the application of radiomics based on cardiac magnetic resonance imaging, cardiac computed tomography and TTE in diagnosing several myocardial abnormalities, such as myocardial infarction, myocarditis, cardiac amyloidosis, and hypertrophic cardiomyopathy (HCM). In this study, we explored the possibility of using myocardial texture features in differentiating HCM, hypertensive heart disease (HHD) and uremic cardiomyopathy (UCM) based on echocardiography. To our knowledge, this was the first study to explore TTE myocardial texture analysis for multiple LVH etiology differentiation.

Methods: TTE images were reviewed retrospectively from January 2018 to collect 50 cases for each group of HHD, HCM and UCM. The apical four chamber view was retrieved. Seventeen first-order statistics and 60 gray level co-occurrence matrix (GLCM) features were extracted for statistics and classification test by support vector machine (SVM).

Results: Of all the parameters, entropy of brightness (EtBrt), standard deviation (Std), coefficient of variation $(\mathrm{CoV})$, skewness (Skew), contrast7 (Cont7) and homogeneity5 (Hm5) were found statistically significant among the three groups $($ all $\mathrm{P}<0.05)$ and with acceptable reproducibility (intraobserver and interobserver ICC >0.50). As a result, HCM showed the most homogeneous myocardial texture, and was significantly different from HHD and UCM (all six features: $\mathrm{P} \leq 0.005$ ). HHD appeared slightly more homogeneous than UCM, as only EtBrt and $\mathrm{CoV}$ were significant $(\mathrm{P}=0.011$ and $\mathrm{P}=0.008)$. According to higher areas under the receiver operating characteristic curve (AUC) (>0.50), EtBrt, Std, and CoV were selected for test of classification as a combination of features. The AUC derived from SVM model was slightly improved compared with those of EtBrt, Std and CoV individually.

Conclusions: AI-based myocardial texture analysis using ultrasonic images may be a potential approach to aiding $\mathrm{LVH}$ etiology differentiation.

Keywords: Left ventricular hypertrophy (LVH); echocardiography; myocardium; artificial intelligence (AI); texture

Submitted Jun 22, 2020. Accepted for publication Oct 30, 2020.

doi: 10.21037/atm-20-4891

View this article at: http://dx.doi.org/10.21037/atm-20-4891 


\section{Introduction}

Left ventricular hypertrophy (LVH) is a common clinical finding related to adverse cardiovascular outcomes, such as myocardial ischemia, arrythmia, congestive heart failure and sudden cardiac death $(1,2)$. Main etiologies are hypertension, hypertrophic cardiomyopathy (HCM), aortic valve stenosis, uremia, and cardiac amyloidosis. Other less common causes include myocarditis, cardiac sarcoidosis, and a few genetic disorders such as Anderson-Fabry disease, Danon disease, mitochondrial cardiomyopathies and more. Transthoracic echocardiography (TTE) plays an important role in LVH diagnosis. It not only provides a comprehensive evaluation of LV morphology and functions, but also detects concurrent structural abnormalities. These information may lead to a definite etiology in a subset of patients, but not necessarily enough in the others for lack of specificity $(3,4)$. Myocardial texture is largely neglected in conventional TTE, because it is usually too subtle, non-specific, and difficult to rate or quantify based on human visual observation. Artificial intelligence $(\mathrm{AI})$ has been developing rapidly in recent years. Previous researchers have reported the application of radiomics and AI-based algorithms from cardiac magnetic imaging (CMR), cardiac computed tomography (CT) and echocardiography data in several myocardial diseases (5-9). However, none has been devoted to multiple LVH etiology differentiation based on echocardiography. Compared with CMR and cardiac CT, TTE is more convenient, costeffective, and commonly available in community hospitals. We hypothesized that the disparities in $\mathrm{LVH}$ histological changes from variant etiologies would lead to different myocardial textures in the echocardiographic image, and the computer might be able to recognize them by quantification and algorithms. Specifically, this study aimed to investigate myocardial texture in hypertensive heart disease (HHD), HCM and uremic cardiomyopathy (UCM) through radiomics, and test the features with an AI-based classifier. We present the following article in accordance with the STARD reporting checklist (available at http://dx.doi. org/10.21037/atm-20-4891).

\section{Methods}

The study was conducted in accordance with the Declaration of Helsinki (as revised in 2013). The study was approved by the Medical Research Ethics Committee of Shanghai East Hospital (No. 2020-028) and individual consent for this retrospective analysis was waived.

\section{Study population}

Study population were recruited retrospectively as shown in the flow chart in Figure 1. Case collection was initiated from the Ultrasound Report System of Shanghai East Hospital by searching the key word "hypertrophy" or "hypertrophic". The date of examination was set from January 1, 2018, with an open end of time-point depending on the number of recruited cases. Next, echocardiographic, and clinical data were carefully reviewed for all the candidates consecutively, until 50 cases for each group were obtained. LVH was defined as left ventricular mass index (LVMI) $>115 \mathrm{~g} / \mathrm{m}^{2}$ in male, LVMI $>95 \mathrm{~g} / \mathrm{m}^{2}$ in female, and the greatest left ventricular (LV) wall thickness $>13 \mathrm{~mm}(10)$. HHD was diagnosed for LVH patients with a history of arterial hypertension, and without a familial history of HCM or other abnormal loading conditions. HCM was diagnosed as the greatest $L V$ wall thickness $\geq 15 \mathrm{~mm}$ that is not explained solely by loading conditions (11). Of note, obstructive HCM (HOCM, diagnosed as pressure gradient $\geq 30 \mathrm{mmHg}$ ) was eliminated from the study to avoid mixture. UCM was defined as LVH presentation in patients with chronic endstage renal disease (ESRD), with the glomerular filtration rate $(\mathrm{GFR})<15 \mathrm{~mL} / \mathrm{min} / 1.73 \mathrm{~m}^{2}$. Exclusion criteria include ambiguous etiologies, multiple etiologies (except UCM with hypertension), coronary heart disease, aortic valve stenosis, moderate or severe valvular regurgitation, congenital heart disease, cardiac amyloidosis, diabetes, athletic hearts, and cases with inadequate image qualities. UCM with hypertension were not excluded because all the UCM group members had arterial hypertension secondary to chronic renal failure.

\section{Echocardiography}

TTE had all been performed following the 2015 version of Recommendations of cardiac quantification in adults by the American Society of Echocardiography (ASE) (10). Multiple operators were involved, including novice and experienced echocardiographers. Ultrasound equipment used were GE Vivid E9 (transducer M5S), GE Vivid7 (transducer M4S), Philips IE33 (transducer S5-1) and Philips EPIQ7C (transducer S5-1 and X5-1) systems. Standard apical four-chamber (A4ch) view of the end-diastolic frame was retrieved for analysis. 


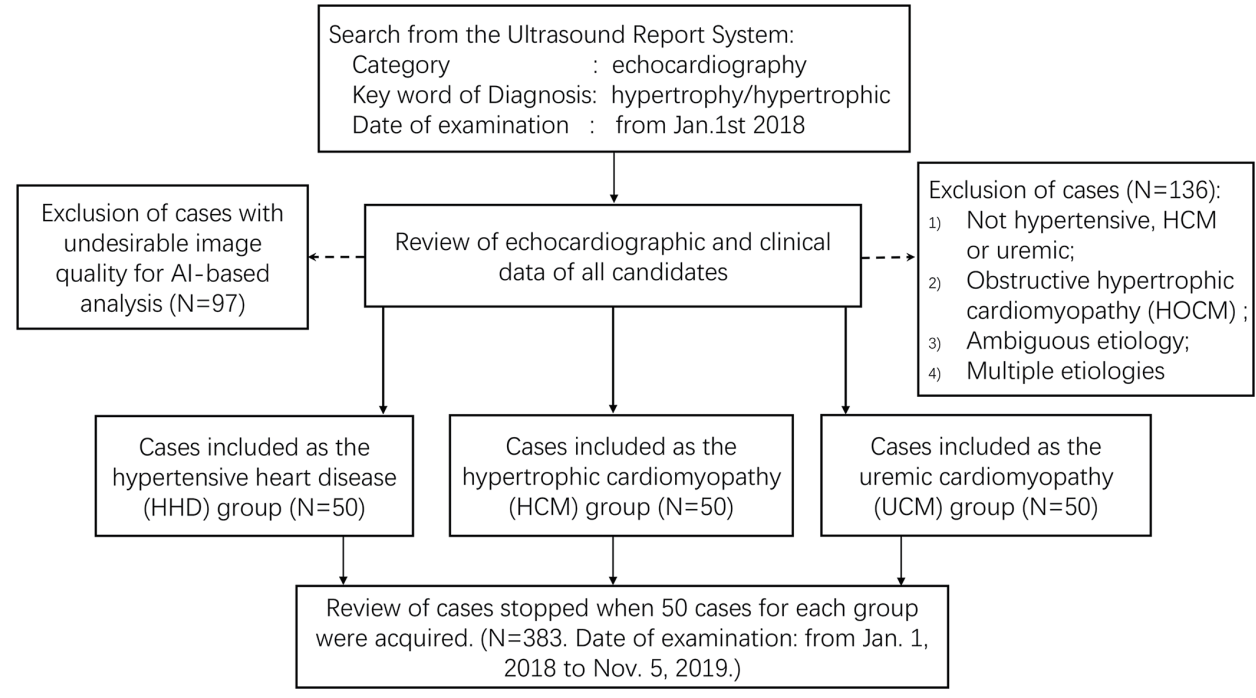

Figure 1 Flow chart of the enrollment procedure.

\section{Extraction of myocardial texture features}

Firstly, a well visualized region of the interventricular septum (IVS) was manually outlined as the region of interest (ROI) by an experienced echocardiographer as shown in Figure 2. The mask image of the ROI was obtained through binarization processing. Then the texture features were extracted, including first-order statistics and gray level cooccurrence matrix (GLCM) features.

In this research, main first-order statistics features were mean (IMean), median (IMedia), standard deviation (Std), coefficient of variation $(\mathrm{CoV})$, skewness (Skew), kurtosis (Kurt), histogram entropy (EtHis) and brightness entropy (EtBrt) of the pixel grayscales within the ROI. More details are available in Appendix 1.

The second category GLCM features were aimed to describe the texture by analyzing spatial correlations of the grayscale. Unlike first-order statistics features, GLCM not only focuses on overall grayscale amplitudes of the pixels, but also studies their spatial correlation characteristics (12). In GLCM analysis, the 256 levels of grayscale were simplified to eight to reduce computation, resulting in $8 \times 8$ grayscale cooccurrence matrix $G(i, j)(I=1,2, \ldots, 8 ; j=1,2$, $\ldots, 8)$. Then the matrix of probability $p(i, j)$ was obtained through normalization of $G(i, j)$. The distance $(d)$ was set as $1,2,3, \ldots, 15$ pixels, and the direction $\theta$ was $0,45,90$ and 135 degrees. Then the texture features of distance $d$ in four directions were averaged and regarded as the result.

There were four types of GLCM features used in our research-energy (E), contrast (Cont), entropy (Et) and homogeneity $(\mathrm{Hm})$. Heterogeneous and rough texture presents high values of energy, contrast and entropy, and a low value of homogeneity. Each type contains 15 values $(d=$ $1,2,3, \ldots, 15$ pixels), making a total of 60 GLCM featuresE1 to E15, Cont1 to Cont15, Et1 to Et15, and Hm1 to $\mathrm{Hm} 15$. The interested reader can find more information, including mathematical equations in Appendix 1.

Intraobserver and interobserver variability was tested via repeated ROI drawing by the same echocardiographer, and another echocardiographer who was a novice.

\section{Statistical analysis}

Statistical analyses were performed to select potentially useful textures features for differentiation. Continuous variables were described as mean $\pm \mathrm{SD}$. Comparisons of continuous variables were performed with one-way ANOVA among three groups. Between any two groups, unpaired $t$ test was used for normal distributed parameters, and KW test for non-normal distributed parameters. $\mathrm{P}$ value $<0.05$ was regarded as statistically significant. The classification threshold for each significant parameter was determined when Youden index (YI) was the highest, and diagnostic sensitivity, specificity, accuracy, and areas under the receiver operating characteristic curve (AUC) were calculated. Next, interobserver and intraobserver variability were tested using intra-class correlation coefficient (ICC). Features with AUCs $>0.70$ and ICC $>0.50$ were then considered as candidates for the subsequent procedure. 

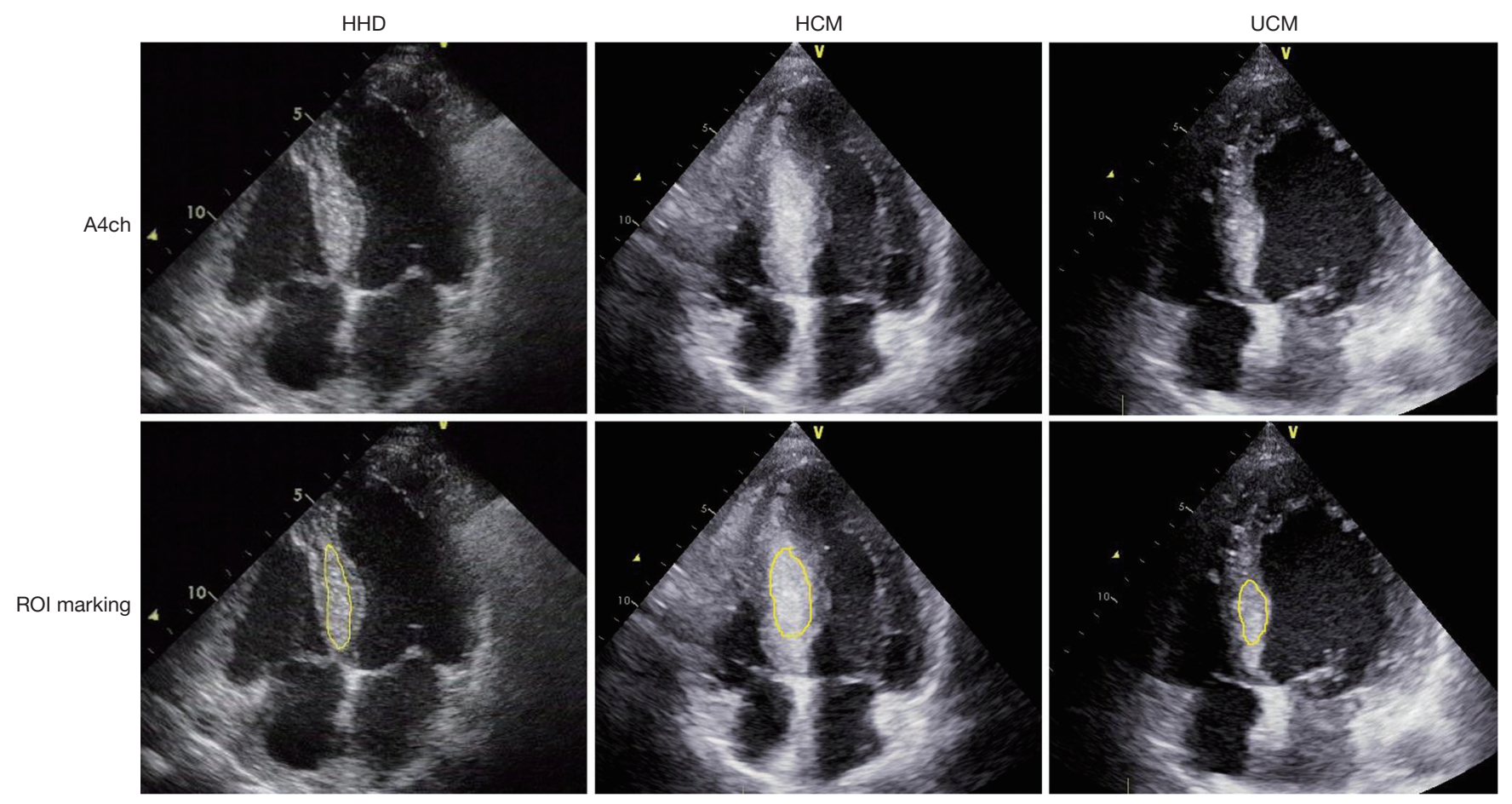

Figure 2 Echocardiographic images of the apical four-chamber (A4ch) view and marking of the hypertrophied ventricular septum as region of interest (ROI). HHD, hypertensive heart disease; HCM, hypertrophic cardiomyopathy; UCM, uremic cardiomyopathy.

\section{Validation of classification}

The selected texture features above were tested for classification by supportive vector machine (SVM), a classifier using supervised learning. Radial basis function (RBF) was applied, and dataset was divided as 6:4 for training and validation. Sensitivity, specificity, accuracy, YI, and AUC were generated for classification of each group from the other two, and between any two groups.

\section{Results}

\section{Patient characteristics and echocardiography}

Patient demography and echocardiographic parameters are listed in Table 1. The HCM group showed the greatest IVS thickness, greatest IVS/LV posterior wall (LVPW) thickness ratio, and smallest LV end-diastolic diameter (LVEDd). UCM group had the thickest LVPW, largest LVEDd and lowest $\mathrm{LV}$ ejection fraction (LVEF). The $\mathrm{P}$ values by oneway ANOVA were all $<0.05$ for $L V$ quantification, except E/e' ratio. However, the UCM group showed a higher E/e' ratio level than $\mathrm{HHD}$, implying poorer $\mathrm{LV}$ diastolic function. Of note, atrial fibrillation (AF) cases were excluded in the E/e' analysis.

\section{Statistics of texture features}

Among the 77 texture features, EtHis, EtBrt, Std, CoV, Skew, Cont7, E11, Hm5 and Et 3 showed relatively better statistical significance, as listed in Table 2. HCM appeared significantly more homogeneous $\mathrm{LV}$ wall than HHD and UCM (all nine features: $\mathrm{P} \leq 0.005$ ). HHD was more homogeneous than UCM, however, significant difference was only yielded in EtBrt and $\mathrm{CoV}(\mathrm{P}=0.011$ and $\mathrm{P}=0.008)$. Accordingly, AUCs of EtBrt and $\mathrm{CoV}$ were also larger than the others, as stated in Table 3 (HCM $v s$. other groups: $\mathrm{AUC}_{\mathrm{EtBrt}}=0.87, \mathrm{AUC}_{\mathrm{CoV}}=0.87$; HHD vs. $\left.\mathrm{UCM}: \mathrm{AUC}_{\mathrm{EtBrt}}=0.65, \mathrm{AUC}_{\mathrm{CoV}}=0.66\right)$. EtHis and Std also showed relatively higher AUC ( $>0.70$ for HCM $v s$. other groups). More details about the cutoff values, sensitivity, specificity, accuracy, YI and AUCs can be found in Appendix 1.

Interobserver and intraobserver variability were assessed with ICC and shown in Table 4. EtHis, E11 and Et3 presented undesirable consistency (interobserver ICC $<0.50$ ) and were excluded in the subsequent experiment. 
Table 1 Patient characteristics and routine echocardiography

\begin{tabular}{|c|c|c|c|c|c|}
\hline Characteristics & $\mathrm{HHD}$ & $\mathrm{HCM}$ & UCM & \multicolumn{2}{|c|}{ One-way ANOVA } \\
\hline No. of cases & $\mathrm{n}=50$ & $n=50$ & $n=50$ & & \\
\hline Age (year) & $64.8 \pm 13.0$ & $54.3 \pm 14.0$ & $61.1 \pm 14.5$ & 7.535 & 0.001 \\
\hline Female & $7(14 \%)$ & $13(26 \%)$ & $11(22 \%)$ & $\chi^{2}=2.277$ & 0.320 \\
\hline IVS thickness (mm) & $13.1 \pm 0.9$ & $19.9 \pm 4.1$ & $13.4 \pm 1.4$ & 110.736 & 0.000 \\
\hline LVPW thickness (mm) & $10.1 \pm 1.5$ & $10.7 \pm 2.6$ & $11.1 \pm 1.7$ & 3.302 & 0.040 \\
\hline IVS/LVPW ratio & $1.32 \pm 0.19$ & $1.95 \pm 0.56$ & $1.32 \pm 0.19$ & 58.597 & 0.000 \\
\hline LVEDd (mm) & $47.4 \pm 4.3$ & $44.6 \pm 5.2$ & $51.0 \pm 6.8$ & 16.590 & 0.000 \\
\hline
\end{tabular}

HHD, hypertensive heart disease; HCM, hypertrophic cardiomyopathy; UCM, uremic cardiomyopathy; IVS, interventricular septum; LVPW, left ventricular posterior wall; LVEDd, left ventricular end-diastolic diameter; LVEF, left ventricular ejection fraction; E, early peak velocity of trans-mitral LV filling; e', diastolic early peak tissue velocity at the mitral annulus.

Table 2 Myocardial texture features of HHD, HCM and UCM (mean \pm Std)

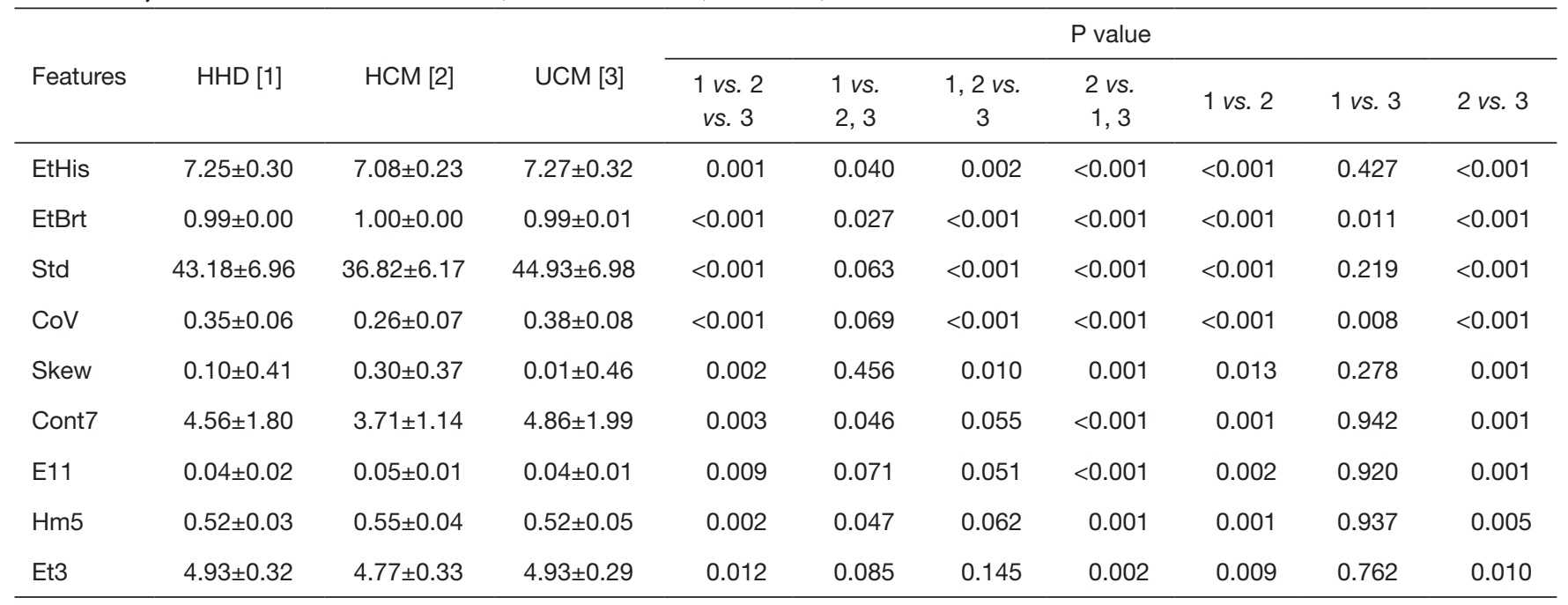

HHD, hypertensive heart disease, is marked as "1" in P value columns; HCM, hypertrophic cardiomyopathy, is marked as " 2 " in P value columns; UCM, uremic cardiomyopathy, is marked as " 3 " in P value columns.

\section{Classification by SVM}

EtBrt, Std and $\mathrm{CoV}$ were remained eventually for validation of classification. The AUC for HHD vs. HCM and UCM reached 0.70 , which was markedly improved than any individual texture feature. The AUC for HHD vs. HCM, and HHD vs. UCM were also slightly improved (0.91 and
0.68). Details of diagnostic accuracy, sensitivity, specificity, and AUC can be found in Table 5 .

\section{Discussion}

$\mathrm{LVH}$ is a common outcome in several clinical conditions, among which hypertension, HCM and chronic renal 
Table 3 The AUCs of texture features

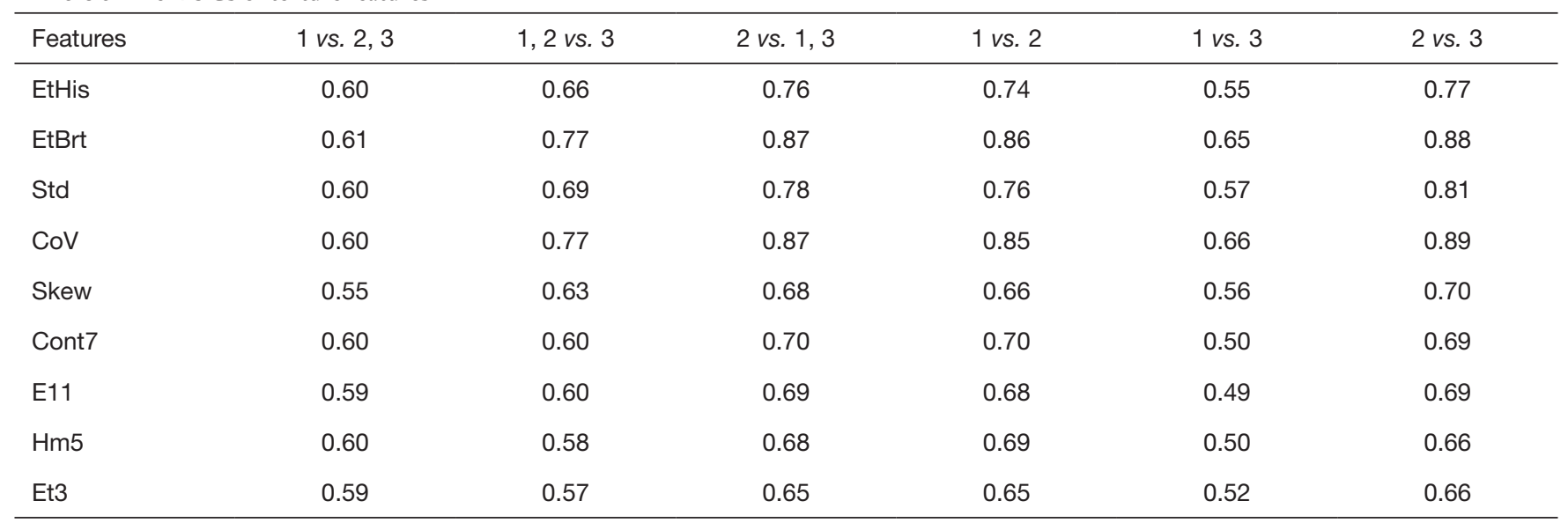

The three groups are marked as "1", "2" and "3" for short in the table. Group 1 stands for hypertensive heart disease, group 2 for hypertrophic cardiomyopathy, and group 3 for uremic cardiomyopathy. AUC, areas under the receiver operating characteristic curve.

Table 4 Interobserver and intraobserver variability of texture features

\begin{tabular}{lcc}
\hline Features & Interobserver ICC & Intraobservoer ICC \\
\hline EtHis & 0.249 & 0.269 \\
EtBrt & 0.734 & 0.707 \\
Std & 0.755 & 0.693 \\
CoV & 0.748 & 0.794 \\
Skew & 0.833 & 0.847 \\
Cont7 & 0.594 & 0.544 \\
E11 & 0.444 & 0.468 \\
Hm5 & 0.613 & 0.743 \\
Et3 & 0.417 & 0.659 \\
\hline
\end{tabular}

ICC, intraclass correlation coefficient. failure are some of the most common etiologies. TTE can evaluate cardiac morphology and function in $\mathrm{LVH}$, which may lead to a definite cause in some cases. For example, a highly asymmetrical pattern of septal hypertrophy or LV apical hypertrophy suggests HCM (11). However, there are still large numbers of nonspecific cases. HCM may also present symmetrical hypertrophy, mimicking HHD. On the contrary, HHD may manifest somewhat asymmetrical hypertrophy as well, with the thickest portion at the basal septum, and even with an IVS/LVPW thickness ratio greater than $1.5(12,13)$. The third group, UCM, is usually related to symmetrical LV hypertrophy, and the odds of $\mathrm{LV}$ dilation and $\mathrm{LV}$ ejection fraction (LVEF) decline is increased (14-16). Therefore, it could be challenging to reveal the real cause of LVH, especially for inexperienced

Table 5 Classification results by support vector machine using EtBrt, Std and CoV

\begin{tabular}{llllll}
\hline Classification between groups & Acc & Sen & Spc & Yl & AUC \\
\hline 1 vs. 2,3 & 0.68 & 0.59 & 0.78 & 0.37 & 0.70 \\
1,2 vs. 3 & 0.68 & 0.77 & 0.76 & 0.42 & 0.75 \\
2 vs. 1,3 & 0.78 & 0.84 & 0.75 & 0.59 & 0.87 \\
1 vs. 2 & 0.85 & 0.90 & 0.80 & 0.70 & 0.91 \\
1 vs. 3 & 0.67 & 0.56 & 0.76 & 0.32 & 0.68 \\
2 vs. 3 & 0.85 & 0.78 & 0.91 & 0.68 & 0.86 \\
\hline
\end{tabular}

The three groups are marked as "1", "2" and "3" for short in the table. Group 1 stands for hypertensive heart disease, group 2 for hypertrophic cardiomyopathy, and group 3 for uremic cardiomyopathy. Sen, sensitivity; Spc, specificity; Acc, accuracy; YI, Youden index; AUC, area under the receiver operating characteristic curve. 
echocardiographers. A comprehensive analysis of multiple imaging data and clinical information is required. Advanced imaging techniques like CMR may be needed, and even endomyocardial biopsy and genetic analysis, which are more costly and not commonly available.

Echocardiography is a fundamental, widely used imaging modality in cardiology. It is very effective in evaluating cardiac structures and functions. However, compared with CMR, TTE has very limited value in tissue characterization, the echogenicity and heterogeneity of myocardium is rarely described in echocardiography, because it is not specific in most cases, and very difficult to rate or quantify based on human visual observation. Hence, we proposed myocardial texture analysis by the computer to overcome the limitation, and hopefully improve TTE diagnostic power. According to our results, different $\mathrm{LVH}$ groups did appear different myocardial texture features in TTE, and they could be identified by the computer. Previous researchers have reported the feasibility and usefulness of myocardial texture analysis based on CMR, cardiac CT and echocardiography in several myocardial abnormalities, such as myocardial infarction, myocarditis, cardiac amyloidosis and HCM (5$9,17)$. In this study, we explored TTE myocardial texture analysis in differentiating three specific LVH entitiesHHD, HCM and UCM. It turned out that EtBrt, Std and $\mathrm{CoV}$ had potentially good diagnostic power and reproducibility. The three parameters were then tested with the SVM model, a classifier using supervised learning. The classification results of EtBrt, Std and $\mathrm{CoV}$ as combined was overall improved than any individual feature. The AUC of distinguishing HHD from HCM reached 0.91 (highest AUC of individual features: 0.86), The AUC of separating HHD from the others reached 0.70 (highest AUC of individual features: 0.61). Classifier models based on AI may further improve diagnostic accuracy of the LVH etiology.

As the statistics showed, HCM presented the highest EtBrt, and lowest Std and CoV, which indicated the most homogeneous myocardial texture. UCM appeared the most heterogeneous, but was close to HHD. These findings could be correlated to the different histological changes. Echogenic features of tissues were determined by histological components and the unity of microstructure alignment (17). For instance, collagen tends to attenuate soundwaves more than cardiomyocyte. Microstructures are usually hyperechoic due to numerous acoustic boundaries (17-19), while water is echolucent. Previous literature stated that common pathological changes in
LVH include cardiomyocyte hypertrophy and disarray, expansion of interstitial and perivascular collagen, and vascular thickening (20-22). We hypothesized that different etiologies may lead to different patterns of LV remodeling, with different levels of myocardial hyperplasia, myocardial disarray, and interstitial fibrosis. UCM develops an even more complex histological pattern by multiple factors, including chronic hypertension, anemia, hypervolemia, and mineral metabolic disorders, resulting in hypertrophy, focal dissolve of cardiomyocyte, interstitial fibrosis, myocardial calcification and oxalate deposition, which might explain the most heterogeneous texture (23).

There were a few limitations to our study. Firstly, it was a single center and retrospective study. The results were not generalizable. Inter-operator and inter-equipment variations of image quality could hardly be eliminated. Secondly, the diagnoses were largely established upon clinical and imaging data according to the latest guidelines and expert consensus. The use of histological or genetic evidence was lacking. Thirdly, most HCM cases in the study had an LV wall over $15 \mathrm{~mm}$ thick, and the average maximum thickness was significantly greater than the other two groups. It was possible that the difference in myocardial texture was more related to the severity of hypertrophy, rather than the etiology. The study was preliminary. However, the application of AI in echocardiography is promising. There have been studies on TTE view recognition, image quality assessment, myocardial motion analysis, diagnostic algorithm establishment and more. There may come a day that a robot can replace the human expert entirely, from automated ultrasound scanning to intellectual image interpretation.

\section{Conclusions}

The current study has preliminarily investigated the feasibility of AI-assisted myocardial texture analysis in distinguishing HHD, HCM and UCM based on TTE still images. It turned out that HCM had a markedly more homogenous texture than HHD and UCM. UCM appeared the most heterogeneous texture, but was close to HHD. Within all the parameters, EtBrt and CoV showed the best diagnostic efficacy. Moreover, SVM model using multiple texture features yielded improved diagnostic accuracy than any single feature. The study implied potential value of myocardial texture features in differentiation of $\mathrm{LVH}$ etiologies by echocardiography. It may add some useful 
supplementary information to the routine TTE, and provide a cost-effective approach to aid diagnoses.

\section{Acknowledgments}

Funding: The work is supported by the National Natural Science Foundation of China (Grant No. 81871361, 61671281 and 61911530249), Shanghai East Hospital "leading talent project" (Grant No. DFRC2018024); Important Weak Subject Construction Project of Pudong Health and Family Planning Commission of Shanghai (Grant No. PWZbr2017-09) and New Medicine Postgraduate Innovation Fund Program of Shanghai University.

\section{Footnote}

Reporting Checklist: The authors have completed the STARD reporting checklist. Available at http://dx.doi.org/10.21037/ atm-20-4891

Data Sharing Statement: Available at http://dx.doi. org/10.21037/atm-20-4891

Conflicts of Interest: All authors have completed the ICMJE uniform disclosure form (available at http://dx.doi. org/10.21037/atm-20-4891). The authors have no conflicts of interest to declare.

Ethical Statement: The authors are accountable for all aspects of the work in ensuring that questions related to the accuracy or integrity of any part of the work are appropriately investigated and resolved. The study was conducted in accordance with the Declaration of Helsinki (as revised in 2013). The study was approved by the Medical Research Ethics Committee of Shanghai East Hospital (No. 2020-028) and individual consent for this retrospective analysis was waived.

Open Access Statement: This is an Open Access article distributed in accordance with the Creative Commons Attribution-NonCommercial-NoDerivs 4.0 International License (CC BY-NC-ND 4.0), which permits the noncommercial replication and distribution of the article with the strict proviso that no changes or edits are made and the original work is properly cited (including links to both the formal publication through the relevant DOI and the license). See: https://creativecommons.org/licenses/by-nc-nd/4.0/.

\section{References}

1. Swamy RS, Lang RM. Echocardiographic quantification of left ventricular mass: prognostic implications. Curr Cardiol Rep 2010;12:277-82.

2. Cuspidi C, Facchetti R, Bombelli M, et al. Risk of mortality in relation to an updated classification of left ventricular geometric abnormalities in a general population: the Pamela study. J Hypertens 2015;33:2133-40.

3. Weidemann F, Niemann M, Ertl G, et al. The different faces of echocardiographic left ventricular hypertrophy: clues to the etiology. J Am Soc Echocardiogr 2010;23:793-801.

4. Angeli F, Reboldi G, Verdecchia P. Echocardiographic left ventricular hypertrophy: implications for clinicians. J Hypertens 2012;30:2279-84.

5. Schofield R, Ganeshan B, Fontana M, et al. Texture analysis of cardiovascular magnetic resonance cine images differentiates aetiologies of left ventricular hypertrophy. Clin Radiol 2019;74:140-9.

6. Hassani C, Saremi F, Varghese BA, et al. Myocardial Radiomics in Cardiac MRI. AJR Am J Roentgenol 2020;214:536-45.

7. Mannil M, von Spiczak J, Manka R, et al. Texture Analysis and Machine Learning for Detecting Myocardial Infarction in Noncontrast Low-Dose Computed Tomography: Unveiling the Invisible. Invest Radiol 2018;53:338-43.

8. Kagiyama N, Shrestha S, Cho JS, et al. A low-cost texturebased pipeline for predicting myocardial tissue remodeling and fibrosis using cardiac ultrasound. EBioMedicine 2020;54:102726.

9. Kerut EK, Given M, Giles TD. Review of methods for texture analysis of myocardium from echocardiographic images: A means of tissue characterization. Echocardiography 2003;20:727-36.

10. Lang RM, Badano LP, Mor-Avi V, et al. Recommendations for Cardiac Chamber Quantification by Echocardiography in Adults: An Update from the American Society of Echocardiography and the European Association of Cardiovascular Imaging. J Am Soc Echocardiogr 2015;28:1-39.e14.

11. Authors/Task Force members; Elliott PM, Anastasakis A, et al. 2014 ESC Guidelines on diagnosis and management of hypertrophic cardiomyopathy. Eur Heart J 2014;35:2733-79.

12. Rodrigues JC, Amadu AM, Dastidar AG, et al. Prevalence and predictors of asymmetric hypertensive heart disease: insights from cardiac and aortic function with 
cardiovascular magnetic resonance. Eur Heart J Cardiovasc Imaging 2016;17:1405-13.

13. Gaudron PD, Liu D, Scholz F, et al. The septal bulge--an early echocardiographic sign in hypertensive heart disease. J Am Soc Hypertens 2016;10:70-80.

14. Arnold R, Schwendinger D, Jung S, et al. Left ventricular mass and systolic function in children with chronic kidney disease-comparing echocardiography with cardiac magnetic resonance imaging. Pediatr Nephrol 2016;31:255-65.

15. Levin A. Anemia and left ventricular hypertrophy in chronic kidney disease populations: A review of the current state of knowledge. Kidney Int Suppl 2002;(80):35-8.

16. Wanner C, Amann K, Shoji T. The heart and vascular system in dialysis. Lancet 2016;388:276-84.

17. Pinamonti B, Picano E, Ferdeghini EM, et al. Quantitative texture analysis in two-dimensional echocardiography: Application to the diagnosis of myocardial amyloidosis. J Am Coll Cardiol 1989;14:666-71.

Cite this article as: Yu F, Huang H, Yu Q, Ma Y, Zhang Q, Zhang B. Artificial intelligence-based myocardial texture analysis in etiological differentiation of left ventricular hypertrophy. Ann Transl Med 2021;9(2):108. doi: 10.21037/ atm-20-4891
18. Skorton DJ, Collins SM, Woskoff SD, et al. Range and azimuth-dependent variability of image texture in twodimensional echocardiograms. Circulation 1983;68:834-40.

19. Smith SW, Wagner RF. Ultrasound speckle size and lesion signal to noise ratio: Verification of theory. Ultrason Imaging 1984;6:174-80.

20. Marian AJ, Braunwald E. Hypertrophic Cardiomyopathy: Genetics, Pathogenesis, Clinical Manifestations, Diagnosis, and Therapy. Circ Res 2017;121:749-70.

21. Ariga R, Tunnicliffe EM, Manohar SG, et al. Identification of Myocardial Disarray in Patients With Hypertrophic Cardiomyopathy and Ventricular Arrhythmias. J Am Coll Cardiol 2019;73:2493-502.

22. Izumaru K, Hata J, Nakano T, et al. Reduced Estimated GFR and Cardiac Remodeling: A Population-Based Autopsy Study. Am J Kidney Dis 2019;74:373-81.

23. Moe SM. Calcium as a Cardiovascular Toxin in CKDMBD. Bone 2017;100:94-99. 


\section{Supplementary methods}

\section{Myocardial texture features}

Two categories of texture features were investigated in the research, namely first-order statistics features and gray level co-occurrence matrix (GLCM) features.

\section{First-order statistics}

First-order statistics features are a series of characteristics that describe the general distribution of pixel brightness within the region of interest (ROI) and the comparison with control areas. Seventeen features were involved in our study, as listed in Table S1.

Histogram entropy (EtHis) and brightness entropy (EtBrt) are two important features of this category. EtHis represents general characteristics of images. A large EtHis value suggests vagueness and uncertainty of the image. It is derived from the following equation:

$$
\text { EtHis }=-\sum_{i=0}^{255} p_{i} \log _{2}\left(p_{i}\right)
$$

In this equation, $P_{i}$ represents the probability of level $i$ grayscale in the specific image ( $i$ from 0 to 255). There are 256 levels of grayscale, from 0 to 255 .

The equation for another feature EtBrt is as follows:

$$
E t B r t=-\sum_{i=1}^{N} \frac{a_{i} \log _{2} a_{i}}{\log _{2}(N)}
$$

In this equation,

$$
a_{i}=\frac{H_{i}}{\sum_{i=1}^{N} H_{i}}
$$

$\mathrm{N}$ is the total number of pixels within the ROI, $H_{i}$ is the level of grayscale of pixel $i(i \leq \mathrm{N})$, and $a_{i}$ stands for the pixel value after normalization.

\section{GLCM features}

The second category GLCM features were initially proposed by Haralick in 1973. Unlike first-order statistics features, GLCM not only focuses on overall grayscale amplitudes of the pixels, but also their spatial correlations. GLCM means the probability of cooccurrence of two pixels with a distance of $d$ and in the direction of $\theta$. An element of the GLCM $G(i, j ; d, \theta)$ represents the times of occurrence of a certain combination of two pixels. In this study, the 256 levels of grayscale were simplified to eight levels to reduce computation, resulting in $8 \times 8$ grayscale cooccurrence matrix $G(i j)(i=1,2, \ldots, 8 ; j=1,2, \ldots, 8)$. Then the matrix of probability $p(i, j)$ was obtained through normalization of $G(i, j)$. The distance $(d)$ was set as $1,2,3, \ldots, 15$ pixels, and the direction $\theta$ was $0,45,90$ and 135 degrees. Finally, the texture features of distance $d$ in four directions were averaged and regarded as the result.

There were four types of GLCM features studied in our research, namely Energy (E), Contrast (Cont), Entropy (Et) and Homogeneity $(\mathrm{Hm})$. Each type contains 15 values $(d=1$, $2,3, \ldots, 15$ pixels), making a total of 60 features, namely E1 to E15, Cont1 to Cont15, Et1 to Et15, and Hm1 to Hm15.

Energy (E) is also named as angular second moment (ASM). It is the sum of the value of each GLCM element squared:

$$
E=\sum_{i=1}^{8} \sum_{j=1}^{8} p(i, j)^{2}
$$

Energy measures the uniformity and texture roughness of pixel intensity distribution. A larger $\mathrm{E}$ value indicates rough texture.

Contrast (Cont) reflects local variation of grayscale values. Greater Cont indicates greater change in pixel brightness, and higher clarity of the image. The equation to yield Cont is as follows:

$$
\text { Cont }=\sum_{i=1}^{8} \sum_{j=1}^{8}|i-j|^{2} p(i, j)
$$

Entropy (Et) manifests the amount of information within the ROI. It reflects complexity and nonuniformity of the image. A larger Et value suggests greater heterogeneity of the image.

$$
E t=-\sum_{i=1}^{8} \sum_{j=1}^{8} p(i, j) \log _{2} p(i, j)
$$

Homogeneity $(\mathrm{Hm})$ is a feature that measures focal texture changes. It reflects similarity and uniformity of the texture within the image. Greater Hm means less change of the texture.

$$
H m=\sum_{i=1}^{8} \sum_{i=1}^{8} \frac{p(i, j)}{1+|i-j|}
$$

\section{Supplementary results}

\section{Statistics of myocardial texture features}

Among all the textural features, EtHis, EtBrt, Std, CoV, Skew, Cont7, E11, Hm5 and Et 3 showed statistical 
significance in intergroup comparisons. The threshold of classification was determined for these nine features according to Youden Index. The results and diagnostic sensitivity, specificity, accuracy, and AUC were listed in Tables $S 2, S 3, S 4, S 5, S 6, S 7$.

Table S1 First-order statistics features

\begin{tabular}{|c|c|c|}
\hline Features & Features (abbreviations) & Full term/definition \\
\hline \multirow{6}{*}{$\mathrm{ROI}$} & IMedia & Median \\
\hline & Std & Standard deviation \\
\hline & Cov & Coefficient of variation \\
\hline & Kurt & Kurtosis \\
\hline & Ethis & Histogram entropy \\
\hline & EtBrt & Brightness entropy \\
\hline \multirow[t]{7}{*}{$\mathrm{ROI} /$ ctrl ratio } & RImedian & $\mathrm{ROI} / \mathrm{ctrl}$ ratio of median brightness \\
\hline & RImedian_quant|90 & ROI median/ctrl $90 \%$ quantile \\
\hline & RImedian_quant195 & ROI median/ctrl 95\% quantile \\
\hline & RImean_quant|90 & ROI mean/ctrl $90 \%$ quantile \\
\hline & RImean_quant|95 & ROI mean/ctrl 95\% quantile \\
\hline & Rlquant|90 & ROI $90 \%$ quantile/ctrl $90 \%$ quantile \\
\hline & Rlquant|95 & ROI 95\% quantile/ctrl 95\% quantile \\
\hline
\end{tabular}

Ctrl, area of control.

Table S2 Diagnostic accuracy of texture features in differentiating HCM from the others

\begin{tabular}{|c|c|c|c|c|c|c|}
\hline Features & Cutoff & Sen & Spc & Acc & $\mathrm{YI}$ & AUC \\
\hline EtBrt & 0.99 & 0.82 & 0.86 & 0.84 & 0.68 & 0.87 \\
\hline Std & 40.75 & 0.76 & 0.71 & 0.73 & 0.47 & 0.78 \\
\hline CoV & 0.30 & 0.78 & 0.88 & 0.84 & 0.66 & 0.87 \\
\hline Cont7 & 4.19 & 0.74 & 0.63 & 0.67 & 0.37 & 0.70 \\
\hline E11 & 0.04 & 0.64 & 0.69 & 0.67 & 0.33 & 0.69 \\
\hline $\mathrm{Hm} 5$ & 0.54 & 0.62 & 0.70 & 0.67 & 0.32 & 0.68 \\
\hline Et3 & 4.87 & 0.66 & 0.64 & 0.65 & 0.30 & 0.65 \\
\hline
\end{tabular}

HCM, hypertrophic cardiomyopathy; Sen, sensitivity; Spc, specificity; Acc, accuracy; YI, Youden index; AUC, area under the receiver operating characteristic curve. 
Table S3 Diagnostic accuracy of texture features in differentiating HHD from the others

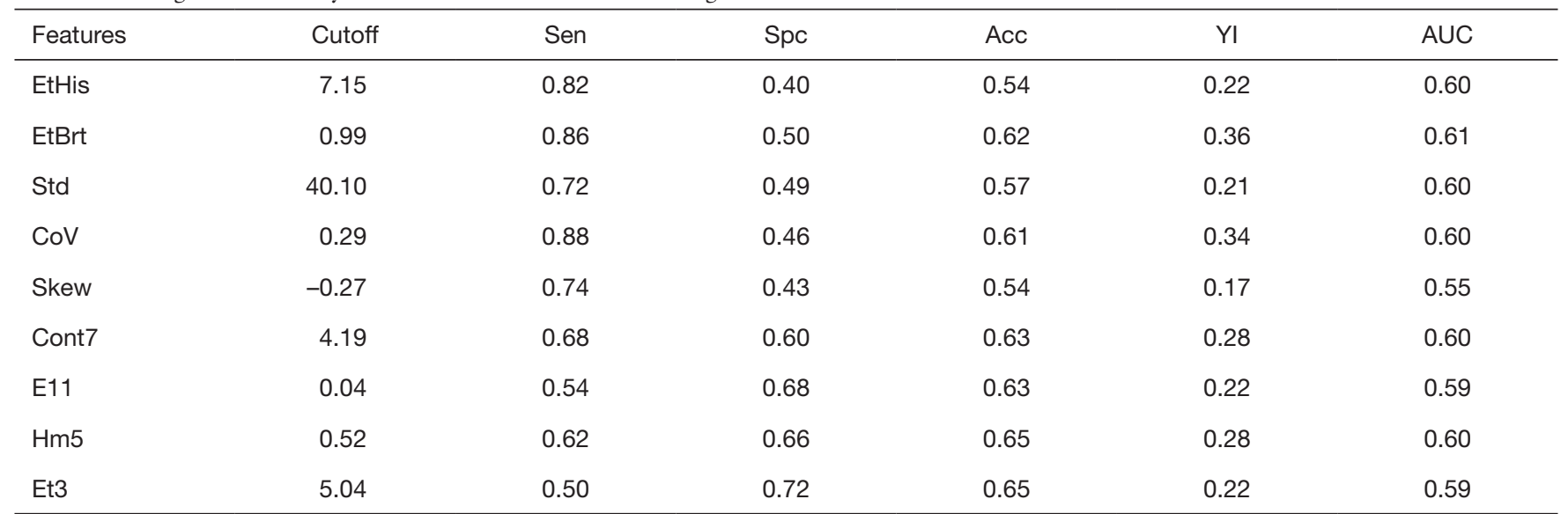

HHD, hypertensive heart disease; Sen, sensitivity; Spc, specificity; Acc, accuracy; YI, Youden index; AUC, area under the receiver operating characteristic curve.

Table S4 Diagnostic accuracy of texture features in differentiating UCM from the others

\begin{tabular}{|c|c|c|c|c|c|c|}
\hline Features & Cutoff & Sen & $\mathrm{Spc}$ & Acc & YI & $A \cup C$ \\
\hline EtBrt & 0.99 & 0.82 & 0.62 & 0.76 & 0.44 & 0.77 \\
\hline Std & 40.93 & 0.55 & 0.75 & 0.61 & 0.30 & 0.69 \\
\hline $\mathrm{CoV}$ & 0.35 & 0.72 & 0.70 & 0.71 & 0.42 & 0.77 \\
\hline Cont7 & 5.26 & 0.86 & 0.32 & 0.69 & 0.18 & 0.60 \\
\hline E11 & 0.04 & 0.52 & 0.72 & 0.59 & 0.24 & 0.60 \\
\hline $\mathrm{Hm} 5$ & 0.50 & 0.85 & 0.36 & 0.69 & 0.21 & 0.58 \\
\hline Et3 & 4.87 & 0.53 & 0.68 & 0.58 & 0.21 & 0.57 \\
\hline
\end{tabular}

UCM, uremic cardiomyopathy; Sen, sensitivity; Spc, specificity; Acc, accuracy; YI, Youden index; AUC, area under the receiver operating characteristic curve.

Table S5 Diagnostic accuracy of texture features in differentiating HHD and HCM

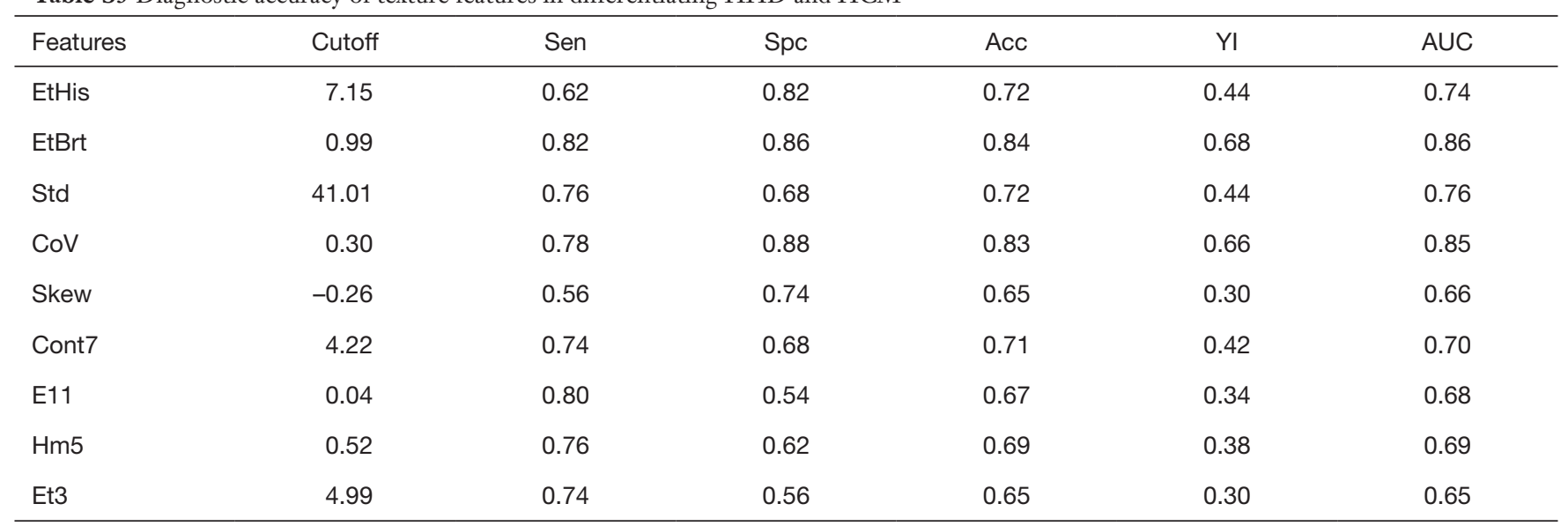

HHD, hypertensive heart disease; HCM, hypertrophic cardiomyopathy; Sen, sensitivity; Spc, specificity; Acc, accuracy; YI, Youden index; AUC, area under the receiver operating characteristic curve. 
Table S6 Diagnostic accuracy of texture features in differentiating HCM and UCM

\begin{tabular}{|c|c|c|c|c|c|c|}
\hline Features & Cutoff & Sen & $\mathrm{Spc}$ & Acc & YI & $A \cup C$ \\
\hline EtHis & 7.19 & 0.66 & 0.81 & 0.73 & 0.47 & 0.77 \\
\hline EtBrt & 0.99 & 0.82 & 0.85 & 0.84 & 0.67 & 0.88 \\
\hline Std & 40.93 & 0.76 & 0.75 & 0.75 & 0.51 & 0.81 \\
\hline CoV & 0.23 & 0.78 & 0.87 & 0.83 & 0.65 & 0.89 \\
\hline Cont7 & 4.19 & 0.74 & 0.57 & 0.66 & 0.31 & 0.69 \\
\hline E11 & 0.04 & 0.64 & 0.72 & 0.68 & 0.36 & 0.69 \\
\hline Hm5 & 0.54 & 0.62 & 0.70 & 0.66 & 0.32 & 0.66 \\
\hline Et3 & 4.87 & 0.66 & 0.68 & 0.67 & 0.34 & 0.66 \\
\hline
\end{tabular}

HCM, hypertrophic cardiomyopathy; UCM, uremic cardiomyopathy; Sen, sensitivity; Spc, specificity; Acc, accuracy; YI, Youden index; AUC, area under the receiver operating characteristic curve.

Table S7 Diagnostic accuracy of texture features in differentiating HHD and UCM

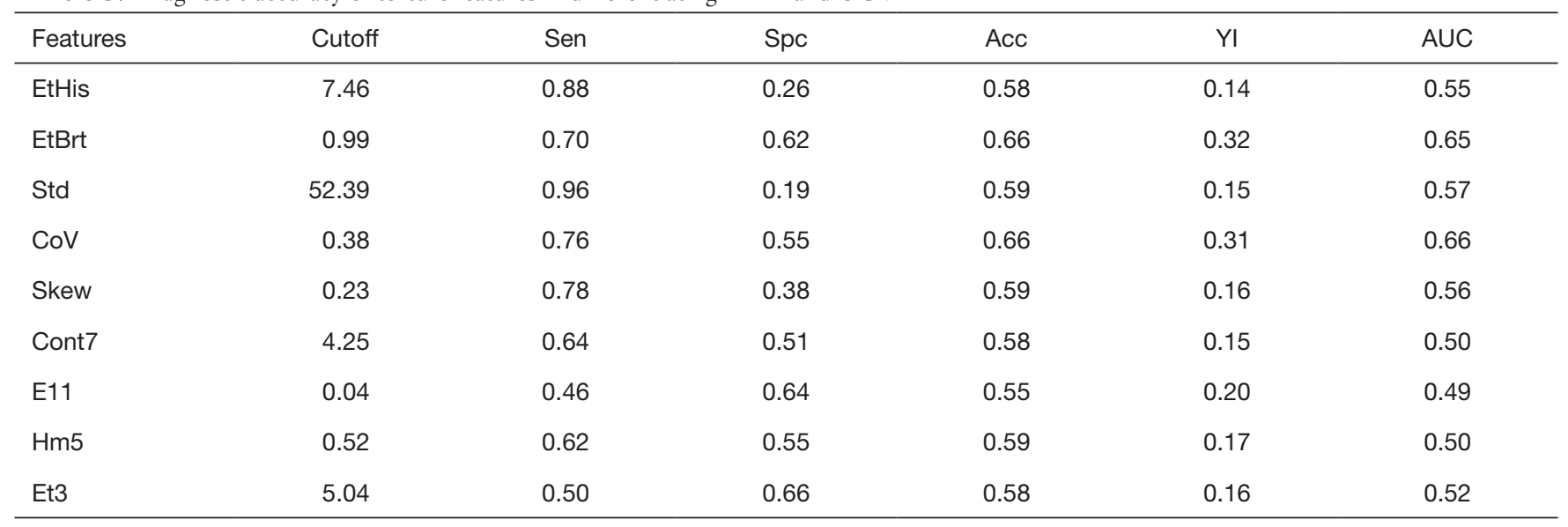

HHD, hypertensive heart disease; UCM, uremic cardiomyopathy; Sen, sensitivity; Spc, specificity; Acc, accuracy; YI, Youden index; AUC, area under the receiver operating characteristic curve. 